\title{
The Higgs trilinear coupling and the scale of new physics
}

\author{
Spencer Chang ${ }^{a, b}$ and Markus A. Luty ${ }^{c}$ \\ ${ }^{a}$ Department of Physics and Institute of Theoretical Science, \\ University of Oregon, Eugene, Oregon 97403, U.S.A. \\ ${ }^{b}$ Department of Physics, National Taiwan University, \\ Taipei, Taiwan 10617, R.O.C. \\ ${ }^{c}$ Center for Quantum Mathematics and Physics (QMAP) \\ University of California, Davis, California 95616, U.S.A. \\ E-mail: chang2@uoregon.edu, maluty@ucdavis.edu
}

\begin{abstract}
We consider modifications of the Higgs potential due to new physics at high energy scales. These upset delicate cancellations predicted by the Standard Model for processes involving Higgs bosons and longitudinal gauge bosons, and lead to a breakdown of the theory at high energies. We focus on modifications of the Higgs trilinear coupling and use the violation of tree-level unitarity as an estimate of the scale where the theory breaks down. We obtain a completely model-independent bound of $\lesssim 13 \mathrm{TeV}$ for an order-1 modification of the trilinear. We argue that this bound can be saturated only in fine-tuned models, and the scale of new physics is likely to be much lower. The most stringent bounds are obtained from amplitudes involving multiparticle states that are not conventional scattering states. Our results show that a future determination of the Higgs cubic coupling can point to a well-defined scale of new physics that can be targeted and explored at future colliders.
\end{abstract}

Keywords: Beyond Standard Model, Higgs Physics

ARXIV EPRINT: 1902.05556 


\section{Contents}

1 Introduction 1

2 Higgs potential modifications and unitarity violation 4

3 Models with a nonstandard Higgs trilinear coupling 13

4 Conclusions $\quad 16$

$\begin{array}{ll}\text { A Unitarity bound on potential interactions } & 17\end{array}$

\section{Introduction}

Many of the couplings of the $125 \mathrm{GeV}$ Higgs boson to gauge bosons and fermions have been measured at the $10 \%$ level and agree with the predictions of the Standard Model [1, 2]. On the other hand, the Higgs potential is very weakly constrained experimentally. If we define $h$ to be the Higgs field measured relative to its vacuum expectation value, the $h^{2}$ term gives the Higgs mass, but the higher order terms in the potential are very weakly constrained. For example, we currently do not know whether the Higgs potential is a double well as predicted by the Standard Model, or a shifted single-well as in models of induced electroweak symmetry breaking [3-6] (see figure 1). Another well motivated theory is the Standard Model with a large, modified Higgs trilinear, giving the strong first order electroweak phase transition as needed for electroweak baryogenesis [7]. Such models can be clearly distinguished by the coefficient of the $h^{3}$ term in the Higgs potential, which can be probed in di-Higgs production. This measurement is difficult due to low rates and large backgrounds. The present limits from the LHC constrain the Higgs trilinear to lie in the range -5 to +12 times the Standard Model value [8-10]. Current studies for the high-luminosity LHC indicate that Higgs pair production can only probe the trilinear coupling at best at the $O(1)$ level [11-13]. Future high energy lepton or hadron colliders are required for a more accurate determination with potential sensitivity at the $10 \%$ level [1416]. In comparison, even at future colliders, triple Higgs production is not sensitive to the Standard Model prediction, but can be sensitive to large enough modifications [17-19]. For a recent review on collider Higgs probes, see [20]. Indirect constraints on the Higgs self interactions have also been studied for precision electroweak observables and loop level corrections to Higgs cross sections (e.g. [21-28]), which also have sensitivity but are more model dependent.

An important motivation for this difficult measurement is that a deviation from the Standard Model prediction for the Higgs cubic coupling is a sign of new fundamental particles and/or interactions beyond those described by the Standard Model. By itself, 


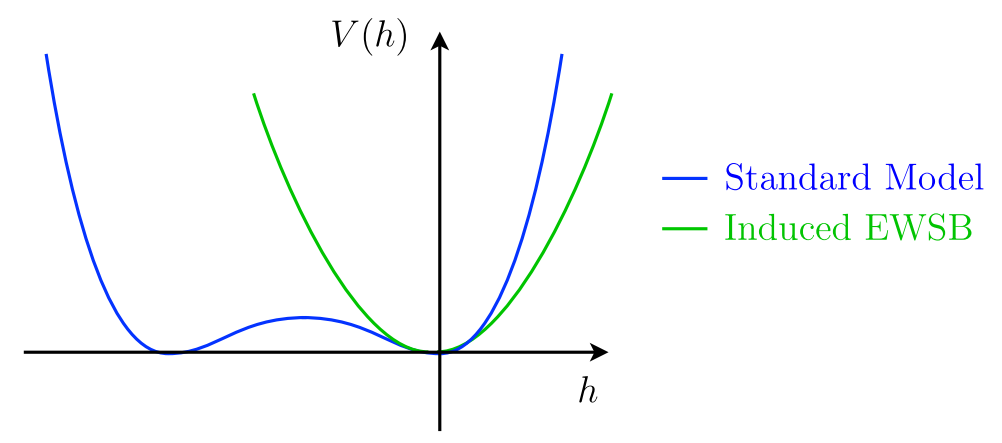

Figure 1. Higgs potentials in the Standard Model and in induced electroweak symmetry breaking.

the deviation in one coupling will not give very much information about what kind of new physics is responsible. However, one model-independent conclusion that can be drawn from such a result is that the mass scale associated with this new physics cannot be arbitrarily large. This is because the Standard Model is the unique perturbatively UV complete theory containing only the experimentally observed elementary particles and interactions. If the new physics that gives rise to the deviation occurs at a mass scale $M$ that is much larger than $m_{h}$, then physics below the scale $M$ can be described by an effective theory with the same degrees of freedom as the Standard Model. This theory will not be UV complete, and will break down at some UV scale, which in turn gives an upper bound on the scale $M$. This bound can be determined entirely from the effective theory, which consists of the Standard Model plus additional local terms that account for the observed deviation from the Standard Model.

In this paper, we give a model-independent estimate of the scale of new physics associated with a deviation in the measured value of the Higgs cubic interaction. The point is that the UV incompleteness of the effective theory that describes the deviation shows up in the violation of tree-level unitarity at high energies. Unitarity is restored order by order in perturbation theory, since the Hamiltonian is Hermitian, but the violation of unitarity at tree-level means that the loop corrections that restore unitarity are order- 1 corrections, i.e. the loop expansion is breaking down and the theory is becoming strongly coupled. We interpret this strong coupling as a sign that new physics is required at or below this scale, since there is no unique way to extrapolate the theory to higher energy scales. This is the standard argument that leads to the use of tree-level unitarity as a diagnostic for the scale of new physics. Note that the scale of strong coupling defined in this way is ambiguous up to $O(1)$ factors; for example loop corrections near the strong coupling scale will give $O(1)$ corrections to tree-level couplings. Nonetheless, tree-level unitarity gives a useful $O(1)$ estimate of the scale where we expect new physics to appear.

This is a variation on a classic success story in particle physics. Long before the discovery of the Higgs boson, Lee, Quigg, and Thacker [29,30] observed that in a theory without a Higgs sector, the scattering of longitudinally polarized $W$ and $Z$ bosons violates tree-level unitarity in the UV, and used this to give a model-independent upper bound on the scale of the Higgs sector. This work has been refined and extended in many ways, see 
for example [31, 32]. These arguments were one of the most important motivations for the energy scale of the LHC, which was in fact successful in discovering the Higgs boson. The present experimental situation is in a sense opposite, in that the minimal theory with the observed particle content can be consistently extrapolated to exponentially large energy scales. But this is only the case if all of the couplings in the theory have the precise values predicted by the Standard Model. Any observed deviation from the predictions of the Standard Model will therefore point to a scale of new physics, just as the existence of massive $W$ and $Z$ bosons pointed to the scale of the Higgs sector.

There are several novel features in our analysis. First, we do not assume that the leading deviations from the Standard Model arise from the leading terms in an effective field theory framework such as SMEFT [33, 34] or HEFT [35] (as reviewed in [36, 37]). These are different parameterizations of the most general physics beyond the Standard Model, assuming no new light particles. They differ only in the power counting rule that determines the relative importance of various contributions. In other words, a given deviation from the SM may appear at different orders in the SMEFT and HEFT expansions, so the truncation of the expansion to a finite number of terms can be different. There is no universally correct expansion; various types of new physics have effective theories with different low-energy expansions (see for example [38]). In this work we will be maximally conservative, and simply assume that a measured deviation in the Higgs cubic coupling could come with any combination of the infinitely many interaction terms that we can add to the effective theory. That is, we allow arbitrary cancellations or conspiracies among these infinitely many terms. We simply maximize the scale of new physics over all possible ways of accounting for the deviation, so our result is completely model-independent.

There are several other innovations in our analysis of a more technical nature that improve upon earlier studies of unitarity violation in many-particle amplitudes [39]. First, we give a simple method of identifying the leading unitarity-violating processes implied by a given local modification of the Standard Model Lagrangian by using the equivalence theorem. Another novelty is that the strongest model independent unitarity bounds on a Higgs trilinear arise from 3 -to-3 processes, and we show that in general the optimal unitarity bounds result from using states other than conventional scattering states.

We comment briefly on some related work. Ref. [39] also studied high-energy unitarity violation in multiparticle processes arising from deviations in the Higgs potential, focusing mainly on the possibility of experimentally observing large $2 \rightarrow n$ processes. Some more recent work along these lines is in ref. [40]. Ref. [41] looked at unitarity violation due to the Higgs trilinear in Higgs-Higgs scattering, which for a sufficiently large deviation, violates unitarity at low energies rather than at high energies. Constraints from perturbative unitarity for $2 \rightarrow 2$ processes in the context of SMEFT were studied in refs. [42, 43], but not for processes induced by new contributions to the Higgs potential. Recent work has also raised the possibility of large non-perturbative effects within the Standard Model related to processes with large multiplicity produced at threshold (for example ref. [44]). The size of these effects is controversial $[45,46]$. In any case, our work involves processes with low multiplicity in the highly boosted regime which are under perturbative control in the Standard Model, but that violate unitarity due to deviations from the Standard Model. 
This paper is organized as follows. In $\S 2$ we analyze the constraints from unitarity violation from arbitrary modifications of the Higgs potential. We show that a generic modification of the Higgs potential leads to unitarity violation near the $\mathrm{TeV}$ scale. We also show that certain unitarity-violating processes are determined completely by the deviation of the Higgs trilinear from the Standard Model prediction, leading to a much more conservative but completely model-independent bound near $13 \mathrm{TeV}$ for an order- 1 deviation in the Higgs trilinear. In $\S 3$ we consider possible models of new physics at high energy scales. We argue that models that saturate the conservative bound require fine-tuning of not only the Higgs mass, but also the Higgs quartic coupling. Our conclusions are in $\S 4$, and an appendix describes a general analysis of unitarity violation from scalar potential terms.

\section{Higgs potential modifications and unitarity violation}

We assume that physics below some UV scale $\Lambda$ can be described by an effective quantum field theory with the particle content of the SM. Any deviations from the SM therefore arise by integrating out states above the scale $\Lambda$, and will result in additional local couplings of the SM fields. As discussed in the introduction, we do not want to make any assumption about the relative importance of the infinitely many possible terms in this Lagrangian. We simply assume that the effective Lagrangian can be written as

$$
\mathcal{L}_{\text {eff }}=\mathcal{L}_{\mathrm{SM}}+\delta \mathcal{L},
$$

where $\delta \mathcal{L}$ is small only in the sense that the effective Lagrangian agrees with experiment. It is not a priori obvious that a framework with this level of generality has any predictive power. We begin by considering the case of modifications of the Higgs potential. To perform a complete analysis, we have to include all allowed Higgs interactions, including interactions with Standard Model gauge bosons and fermions. For example, di-Higgs production via gluon-gluon fusion depends on the Higgs couplings to the top quark and to gluons, as well as the trilinear Higgs coupling (see e.g. [47]). However, the couplings to gluons and top quarks have stronger experimental constraints than the Higgs trilinear. Derivative couplings must also be considered, but they typically give rise to lower unitarity violating scales, as we will discuss at the end of this section.

To write the most general deviation in the Higgs potential, we can write the Lagrangian in unitary gauge where the eaten Nambu-Goldstone bosons are set to zero. In this gauge, the Standard Model Higgs doublet is given by

$$
H=\frac{1}{\sqrt{2}}\left(\begin{array}{c}
0 \\
v+h
\end{array}\right),
$$

where $h$ is the physical Higgs field. We assume that the minimum of the Higgs potential is at $h=0$, i.e. $v$ is the minimum of the full Higgs potential, including any deviations from the SM. The scalar potential is therefore a function of $h$ alone:

$$
V_{\mathrm{eff}}=V_{\mathrm{SM}}(h)+\delta V(h)
$$


where

$$
\begin{aligned}
V_{\mathrm{SM}}(h) & =\frac{1}{2} m_{h}^{2} h^{2}+\frac{m_{h}^{2}}{2 v} h^{3}+\frac{m_{h}^{2}}{8 v^{2}} h^{4}, \\
\delta V(h) & =\sum_{n=3}^{\infty} \frac{\delta \lambda_{n}}{n !} h^{n} .
\end{aligned}
$$

Our focus on general Higgs boson interactions is reminiscent of the Higgs Effective Field Theory (HEFT) approach [35]. Our assumption that $v$ is the true Higgs VEV implies that there are no $O(h)$ terms in $\delta V$. We do not include $O\left(h^{2}\right)$ terms in $\delta V$ because these can be absorbed into a redefinition of $m_{h}$, which is well measured. It is only the cubic and higher terms that represent a true deviation from the SM, as opposed to a change in the value of SM parameters. We have $v=246 \mathrm{GeV}$ and $m_{h}=125 \mathrm{GeV}$ to high accuracy, so the Higgs cubic and quartic couplings are accurately predicted in the SM.

We comment briefly on the role of loop corrections to the Higgs potential. Loops involving heavy particles beyond the Standard Model can be expanded in powers of $h$ about the VEV to give local terms of the form given above. This leaves only loops involving Standard Model fields. These loop corrections are perturbatively small until we get to the scale of tree-level unitarity violation. At that scale, they are expected to be $O(1)$ corrections, so including them would only change the unitarity violating scale by $O(1)$.

Gauge invariance is not manifest in eqs. (2.4), but the potential is the same as a general gauge invariant potential written in terms of the Higgs doublet $H$ when both potentials are expanded around the Higgs VEV. We can simply write the potential as a sum of gauge invariant terms of the form $\left(H^{\dagger} H\right)^{n}$. It is convenient to write this in terms of the variable

$$
Y=H^{\dagger} H-\frac{1}{2} v^{2}
$$

This has vanishing VEV and is linear in $h$, so minimization of the potential is simply the statement that the potential starts with a positive $Y^{2}$ term. The Higgs potential can then be written as

$$
\begin{aligned}
V_{\mathrm{SM}} & =m_{H}^{2} H^{\dagger} H+\lambda\left(H^{\dagger} H\right)^{2}=\lambda Y^{2}+\text { constant } \\
\delta V & =\sum_{n=3}^{\infty} \frac{c_{n}}{n !} Y^{n}
\end{aligned}
$$

where $m_{h}^{2}=2 \lambda v^{2}$. The relation between the couplings $c_{n}$ and $\lambda_{n}$ is easily worked out:

$$
\delta \lambda_{3}=c_{3} v^{3}, \quad \delta \lambda_{4}=c_{4} v^{4}+6 c_{3} v^{2}, \quad \ldots
$$

eqs. (2.4) and (2.6) are two completely equivalent parameterizations of the Higgs potential. We are free to use the parameterization that is more useful for our purposes.

The parameterization eq. (2.6) is the one most commonly used in discussions of new high-scale physics, and it is worth recalling the reason for this. If we make the assumption that the new physics is associated with a large mass scale $M$, and that this physics decouples from electroweak symmetry breaking in the limit where $M$ is large, then we 
expect that the importance of the couplings in the expansion eq. (2.6) will be ordered by dimensional analysis:

$$
c_{n} \sim \frac{1}{M^{2(n-2)}} .
$$

More generally, we can write the most general term in the effective Lagrangian as a sum of local gauge invariant operators, and assign them a power of $M$ by dimensional analysis. Assuming that all coefficients in units of $M$ are the same order, we obtain a predictive truncation of the effective theory, the so-called "Standard Model Effective Field Theory" (SMEFT) [37]. Although this power counting is expected to hold in a large class of models, it is not completely general. For example, operators containing derivatives may be suppressed by a parametrically different scale [48]. Alternatively, the new physics may include heavy particles whose mass comes from electroweak symmetry breaking, which do not decouple at large mass. (Although the precision electroweak $S$ parameter is a nontrivial constraint on such a scenario, it is not cleanly ruled out unless there are many heavy non-decoupling states.) Finally, we may want to allow some of the coefficients to be anomalously small, perhaps because of weak couplings, accidental cancellations, or approximate symmetries.

Our goal is to bound the scale of tree-level unitarity violation associated with deviations from the SM in a completely model-independent way, focusing on the Higgs trilinear. ${ }^{1}$ In doing so, we will not make any assumption about the relative size of the coefficients in the effective theory. It is most convenient for our purposes to use the parameterization in eqs. (2.4), since the new terms in the Lagrangian are in one-to-one correspondence with new effective couplings at the weak scale. Not surprisingly, we will find that tree-level unitarity violation is dominated by amplitudes involving Higgs bosons and longitudinally polarized $W$ and $Z$ bosons $\left(W_{L}\right.$ and $Z_{L}$ ). The equivalence theorem tells us that the scattering amplitudes of $W_{L}$ and $Z_{L}$ at high energies are the same as the scattering amplitudes of the corresponding unphysical Nambu-Goldstone bosons in a general gauge. We can determine the dependence on the unphysical Nambu-Goldstone bosons directly from the potential eq. $(2.4 \mathrm{~b})$ by using the gauge invariant operator

$$
\begin{aligned}
X & =\sqrt{2 H^{\dagger} H}-v \\
& =h+\frac{\vec{G}^{2}}{2(v+h)}-\frac{\vec{G}^{4}}{8(v+h)^{3}}+O\left(\frac{\vec{G}^{6}}{(v+h)^{5}}\right),
\end{aligned}
$$

where $\vec{G}=\left(G_{1}, G_{2}, G_{3}\right)$ are the unphysical Nambu-Goldstone bosons. In unitary gauge we have simply $X=h$, so eq. (2.4b) can be written in a gauge invariant way as

$$
\delta V=\sum_{n=3}^{\infty} \frac{\delta \lambda_{n}}{n !} X^{n} .
$$

\footnotetext{
${ }^{1}$ Previous analyses of the unitarity violation of the Higgs trilinear [41], found that a large trilinear (about seven times the Standard Model value) leads to unitarity violation for the process $h h \rightarrow h h$ near threshold, but with good behavior at high energies. Any new physics that can unitarize this process should therefore be at low energies and within reach of the LHC.
} 
The variable $X$ is not a local operator expanded around $H=0$, but it is a sum of local operators when expanded about the physical VEV, which is what we will do for the rest of the paper. As mentioned earlier, expanding around the VEV also shows why the HEFT and SMEFT frameworks are equivalent, since both lead to a power series in $2 v h+h^{2}+\vec{G}^{2}$.

For example, if we assume that the potential eq. (2.4b) contains only a modification of the $h^{3}$ term, then the gauge invariant form contains terms with arbitrarily high powers of the Higgs and Nambu-Goldstone fields. A particularly simple class is the ones with 2 powers of the Nambu-Goldstone fields [49]:

$$
\delta V \supset \frac{\delta \lambda_{3}}{4 v} h^{2} \vec{G}^{2} \sum_{m=0}^{\infty}\left(-\frac{h}{v}\right)^{m} .
$$

These give scattering amplitudes for the unphysical Goldstone fields that grow with the center of mass energy. By the equivalence theorem these are equal to physical $W_{L}$ and $Z_{L}$ scattering amplitudes at high center of mass energy, and therefore lead to a violation of tree-level unitarity at high energies.

In appendix A we derive unitarity constraints from non-derivative interactions involving many fields. For an interaction of the form

$$
\mathcal{L}_{\text {int }}=\frac{\lambda_{n}}{n_{1} ! \cdots n_{r} !} \phi_{1}^{n_{1}} \phi_{2}^{n_{2}} \cdots \phi_{r}^{n_{r}}
$$

we derive a unitarity bound on the center of mass energy $E_{k}$,

$$
E_{k} \leq 4 \pi\left(\frac{8 \pi \prod_{i=1}^{r} n_{i} !}{\lambda_{n} \prod_{i=1}^{r}\left(\begin{array}{l}
n_{i} \\
k_{i}
\end{array}\right)}\right)^{1 /(n-4)}\left(\frac{(k-1) !(k-2) !(n-k-1) !(n-k-2) !}{\prod_{i=1}^{r} k_{i} !\left(n_{i}-k_{i}\right) !}\right)^{1 /(2 n-8)}
$$

for the process $\phi_{1}^{k_{1}} \cdots \phi_{r}^{k_{r}} \leftrightarrow \phi_{1}^{n_{1}-k_{1}} \cdots \phi_{r}^{n_{r}-k_{r}}$ where we've defined $n \equiv n_{1}+\cdots+n_{r}, k \equiv$ $k_{1}+\cdots+k_{r}$. This general formula automatically takes into account combinatorial factors from Bose statistics. The best bounds come from processes where the fields are equally distributed between the initial and final state, $k_{i}=n_{i} / 2$ for even $n_{i}$. For additional details, see appendix A.

Using the interactions in eq. (2.11) we can now give a unitarity bound for the process $Z_{L} h^{n / 2} \rightarrow Z_{L} h^{n / 2}$ for even $n\left(Z_{L} h^{(n-1) / 2} \rightarrow Z_{L} h^{(n+1) / 2}\right.$ for odd $\left.n\right)$ in terms of the fractional modification of the Higgs trilinear interaction

$$
\delta_{3}=\frac{\delta \lambda_{3}}{\lambda_{3}^{(\mathrm{SM})}}=\frac{v \delta \lambda_{3}}{3 m_{h}^{2}} .
$$

The result is shown in figure 2. The unitarity bounds are strongest for $n \sim 6$ to 22 . Note that we require $E_{\max } \gg \frac{n}{2} m_{h}+m_{Z}$ to justify the use of the equivalence theorem and the use of massless phase space, but this is well satisfied as can be seen in figure 2 . The bounds 




Figure 2. The unitarity violating scale for the interaction $Z_{L}^{2} h^{n}$ as a function of $n$ for different values of $\delta_{3}$. The dashed line shows the threshold energy $\frac{n}{2} m_{h}+m_{Z}$, which compared with the unitarity violating scale shows that $E_{\max }$ is large enough to justify the use of the equivalence theorem and massless phase space. The best limits are $\left(\delta_{3}, n, E_{\max } / \mathrm{TeV}\right)=$ $(0.01,22,5.2),(0.1,18,4.6),(1,12,3.8),(10,6,2.8)$.

are quite strong. For example, for $\delta_{3} \sim 1$ the theory violates unitarity near $4 \mathrm{TeV}$, and even for $\delta_{3} \sim 0.01$ the unitarity violation scale is near $5 \mathrm{TeV}$. The bound is weakly dependent on $\delta_{3}$ because for large multiplicity the dependence on the coupling is reduced, as can be seen from eq. (2.13). These unitarity scales are low enough that they are plausibly within reach of experimental searches at the LHC and future colliders.

The existence of tree-level unitarity violating processes involving $W_{L}$ and $Z_{L}$ can also be understood directly from tree-level Feynman diagrams, without the formalism introduced above. The point is that some of the tree-level diagrams that contribute to these processes involve the Higgs cubic coupling (see for example, figure 3). In the SM, there are cancellations in the high-energy behavior of the amplitude that depend on the Higgs cubic coupling having the SM value. When the cubic coupling deviates from the SM value, this cancellation is absent and the amplitude has harder high-energy behavior. The utility of the formalism discussed here is that it makes it easy to identify the leading high energy behavior in amplitudes involving many initial and final state particles.

Modifying only the $h^{3}$ term may appear to be a reasonable phenomenological model, but we have seen that it makes a dramatic prediction of tree-level unitarity violation at low energy scales. Before concluding that a modification of the cubic coupling implies new physics at such low scales, we must determine whether this conclusion is robust. In fact, it is easy to see that it is not, because there can be cancellations coming from higher order terms of the form $X^{n}$ in eq. (2.10). For example, if the modification consists of only the SMEFT operator $Y^{3}$, we cannot have any terms higher than $h^{6}$, since $Y=v h+\frac{1}{2}\left(h^{2}+\vec{G}^{2}\right)$. (In fact, it is easily checked that $Y=v X+\frac{1}{2} X^{2}$ exactly.) A cubic modification alone on 

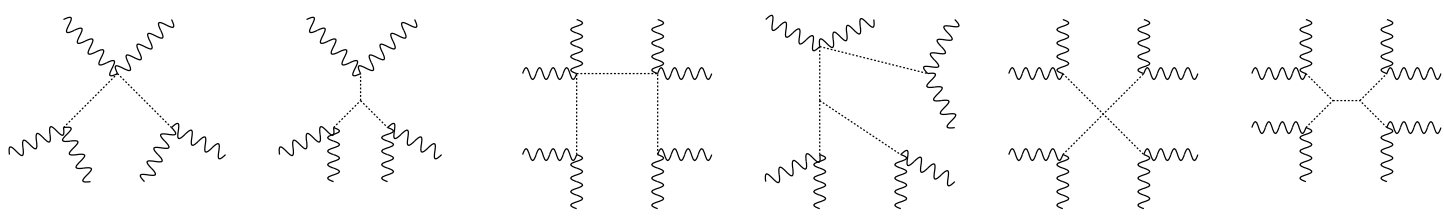

Figure 3. Representative Feynman diagrams for the $Z_{L}^{6}$ and $Z_{L}^{8}$ processes in unitary gauge, demonstrating the dependence on the trilinear and quartic Higgs interactions.

the other hand, involves an infinite series in $Y$,

$$
X^{3}=\left(\sqrt{v^{2}+2 Y}-v\right)^{3}=\frac{Y^{3}}{v^{3}}-\frac{3}{2} \frac{Y^{4}}{v^{5}}+\frac{9}{4} \frac{Y^{5}}{v^{7}}-\frac{7}{2} \frac{Y^{6}}{v^{9}}+\cdots
$$

whose coefficients in units of $v$ do not fall off for higher powers, and it is therefore not surprising that this predicts high multiplicity processes with low scales of unitarity violation. These examples show that the existence of contact interaction terms with many Higgs bosons, which were the origin of the strong unitarity bounds derived above, is not a model-independent consequence of a deviation in the $h^{3}$ coupling.

The lesson is simply that we must consider the most general possible modification of the Higgs potential in order to draw robust conclusions about the high-energy behavior of the theory. To see that there are growing amplitudes for a general modification, we expand the potential eq. (2.10) in powers of $h$ and $\vec{G}$. Powers of $X$ have the structure (see eq. $(2.9))$

$$
\begin{aligned}
X^{3} \sim h^{3} & +\vec{G}^{2}\left(h^{2}+h^{3}+\cdots\right)+\vec{G}^{4}\left(h+h^{2}+\cdots\right)+\vec{G}^{6}(1+h+\cdots) \\
& +\vec{G}^{8}(1+h+\cdots)+\vec{G}^{10}(1+h+\cdots)+\cdots, \\
X^{4} \sim h^{4} & +\vec{G}^{2}\left(h^{3}+h^{4}+\cdots\right)+\vec{G}^{4}\left(h^{2}+h^{3}+\cdots\right)+\vec{G}^{6}\left(h+h^{2}+\cdots\right) \\
& +\vec{G}^{8}(1+h+\cdots)+\vec{G}^{10}(1+h+\cdots)+\cdots, \\
X^{5} \sim h^{5} & +\vec{G}^{2}\left(h^{4}+h^{5}+\cdots\right)+\vec{G}^{4}\left(h^{3}+h^{4}+\cdots\right)+\vec{G}^{6}\left(h^{2}+h+\cdots\right) \\
& +\vec{G}^{8}\left(h+h^{2}+\cdots\right)+\vec{G}^{10}(1+h+\cdots)+\cdots,
\end{aligned}
$$

where we set $v=1$. From this we see that the potential terms

$$
V \supset \frac{m_{h}^{2}}{4 v^{2}}\left(1+3 \delta_{3}\right) \vec{G}^{2} h^{2}+\frac{3 m_{h}^{2}}{8 v^{3}} \delta_{3} \vec{G}^{4} h+\frac{m_{h}^{2}}{16 v^{4}} \delta_{3} \vec{G}^{6},
$$

arise only from the $X^{3}$ term, and are therefore determined by the deviation of the Higgs cubic term in the potential independently of the rest of the Higgs potential. (Note that the interaction $\vec{G}^{2} h^{2}$ is already present in the SM Higgs potential.)

To robustly determine the scale of tree-level unitarity violation implied by a modification of the Higgs cubic, we consider tree-level amplitudes of the fields $h$ and $\vec{G}$ that get contributions from the interaction terms eq. (2.17). We will see below that the strongest bound comes from 3-to-3 processes such as $Z_{L}^{3} \leftrightarrow Z_{L}^{3}$. We will compute this using the equivalence theorem below, but we first consider the calculation in unitary gauge. The tree-level amplitude gets contributions from diagrams like the first two diagrams of figure 3 . The first 
diagram represents 45 different terms obtained by permutations of external legs and vertices, while the second represents 15 . The complexity of this calculation explains why early work on these processes [39] could only be analytically calculated with all of the particle momenta restricted to a common plane and motivates using the much simpler equivalence theorem calculation. At high energies, there are diagrams that are independent of $E$ at high energies, but for the SM value of the Higgs cubic these terms cancel and the amplitude goes as $1 / E^{2}$ at high energy, as required by unitarity. By summing all of these together, one could verify that if the Higgs trilinear interaction is the Standard Model value, the diagrams cancel to achieve the required energy behavior, $1 / E^{2}$, for a unitary six point amplitude. However, if the trilinear is nonstandard, the sum is a constant at high energies that is proportional to $\delta_{3}$.

These results for the six $Z_{L}$ process are much simpler to see using the equivalence theorem. Our potential interactions for the Goldstones do not involve derivatives, so the amplitude's energy dependence comes simply from propagators. Thus, the leading energy dependence is constant and comes from the $\vec{G}^{6}$ contact interaction, which is proportional to $\delta_{3}$. If the Higgs trilinear has the standard value, then there is no six point contact interaction and the amplitude falls off as $1 / E^{2}$ from diagrams with a single propagator. If we now calculate the leading piece, using the results from the appendix, we obtain the unitarity bound

$$
E_{\max } \lesssim \frac{16 \mathrm{TeV}}{\left|\delta_{3}\right|^{1 / 2}}
$$

Bounds for other processes are given in table 1. The strongest five particle process that depends only on the trilinear modification is $h Z_{L}^{2} \leftrightarrow Z_{L}^{2}$, with the bound

$$
E_{\max } \lesssim \frac{94 \mathrm{TeV}}{\left|\delta_{3}\right|}
$$

which gives a stronger bound only for $\left|\delta_{3}\right|>35$, which violates the current LHC constraints on the trilinear.

Optimized bounds for the $\vec{G}^{6}$ interaction can be found by diagonalizing the transition matrix element. Using custodial $S U(() 2)$ symmetry, we can categorize the allowed scattering channels. For $3 G$ 's to $3 G$ 's scattering, there is both a $I=1$ and a $I=3$ channel. As detailed in the appendix, the $I=1$ channel sets the best limit, with

$$
E_{\max } \lesssim \frac{13.4 \mathrm{TeV}}{\left|\delta_{3}\right|^{1 / 2}}
$$

A similar analysis can be done for the $h \vec{G}^{4}$ interaction. Here the allowed channels are $I=0,1$, and 2 . The best bound comes from the $I=0$ channel, with the bound

$$
E_{\max } \lesssim \frac{57.4 \mathrm{TeV}}{\left|\delta_{3}\right|}
$$

These bounds improve a bit upon the channels earlier explored, giving a $\sim 20-40 \%$ reduction in the energy scale for unitarity violation. 


\begin{tabular}{|c|c|}
\hline Process & Unitarity Violating Scale \\
\hline$h^{2} Z_{L} \leftrightarrow h Z_{L}$ & $66.7 \mathrm{TeV} /\left|\delta_{3}-\frac{1}{3} \delta_{4}\right|$ \\
\hline$h Z_{L}^{2} \leftrightarrow Z_{L}^{2}$ & $94.2 \mathrm{TeV} /\left|\delta_{3}\right|$ \\
\hline$h W_{L} Z_{L} \leftrightarrow W_{L} Z_{L}$ & $141 \mathrm{TeV} /\left|\delta_{3}\right|$ \\
\hline$h Z_{L}^{2} \leftrightarrow h Z_{L}^{2}$ & $9.1 \mathrm{TeV} / \sqrt{\left|\delta_{3}-\frac{1}{5} \delta_{4}\right|}$ \\
\hline$h W_{L} Z_{L} \leftrightarrow h W_{L} Z_{L}$ & $11.1 \mathrm{TeV} / \sqrt{\left|\delta_{3}-\frac{1}{5} \delta_{4}\right|}$ \\
\hline$Z_{L}^{3} \leftrightarrow Z_{L}^{3}$ & $15.7 \mathrm{TeV} / \sqrt{\left|\delta_{3}\right|}$ \\
\hline$Z_{L}^{2} W_{L} \leftrightarrow Z_{L}^{2} W_{L}$ & $20.4 \mathrm{TeV} / \sqrt{\left|\delta_{3}\right|}$ \\
\hline$h Z_{L}^{3} \leftrightarrow Z_{L}^{3}$ & $6.8 \mathrm{TeV} /\left|\delta_{3}-\frac{1}{6} \delta_{4}\right|^{\frac{1}{3}}$ \\
\hline$h Z_{L}^{2} W_{L} \leftrightarrow Z_{L}^{2} W_{L}$ & $8.0 \mathrm{TeV} /\left|\delta_{3}-\frac{1}{6} \delta_{4}\right|^{\frac{1}{3}}$ \\
\hline$Z_{L}^{4} \leftrightarrow Z_{L}^{4}$ & $6.1 \mathrm{TeV} /\left|\delta_{3}-\frac{1}{6} \delta_{4}\right|^{\frac{1}{4}}$ \\
\hline
\end{tabular}

Table 1. Unitarity violating amplitudes that only depend on the trilinear and quartic Higgs modifications.

Let us consider what happens if we also include the effects of the quartic interaction. From eq. (2.16), we see that the new terms which depend only on the $h^{3}$ and $h^{4}$ modification are

$$
\begin{aligned}
V \supset & \frac{m_{h}^{2}}{8 v^{2}}\left(1+\delta_{4}\right) h^{4}+\frac{m_{h}^{2}}{4 v^{3}}\left(\delta_{4}-3 \delta_{3}\right) h^{3} \vec{G}^{2}+\frac{3 m_{h}^{2}}{16 v^{4}}\left(\delta_{4}-5 \delta_{3}\right) h^{2} \vec{G}^{4} \\
& +\frac{m_{h}^{2}}{16 v^{5}}\left(\delta_{4}-6 \delta_{3}\right) h \vec{G}^{6}+\frac{m_{h}^{2}}{128 v^{6}}\left(\delta_{4}-6 \delta_{3}\right) \vec{G}^{8} .
\end{aligned}
$$

These can give stronger unitarity bounds, depending on the value of the deviation in the Higgs quartic interaction, see table 1 . For example, the process $Z_{L}^{4} \leftrightarrow Z_{L}^{4}$, which would normally require evaluation of several diagrams as shown in figure 3 , can be easily analyzed with the equivalence theorem to give a unitarity bound

$$
E \lesssim \frac{6.1 \mathrm{TeV}}{\left|\delta_{3}-\frac{1}{6} \delta_{4}\right|^{\frac{1}{4}}},
$$

where we define the fractional quartic coupling deviation

$$
\delta_{4}=\frac{\delta \lambda_{4}}{\lambda_{4}^{(\mathrm{SM})}}=\frac{v^{2} \delta \lambda_{4}}{3 m_{h}^{2}} .
$$

Eq. (2.23) is the unitarity bound that arises from a single insertion of the $\vec{G}^{8}$ contact term that arises from the $X^{3}$ and $X^{4}$ terms in the effective Higgs potential. There are also unitarity-violating contributions to the $Z_{L}^{4} \leftrightarrow Z_{L}^{4}$ amplitude from tree-level diagrams with internal lines, but these are parametrically smaller for $\delta_{3} \sim \delta_{4} \lesssim 1$. For example, there is a contribution with two insertions of the $h \vec{G}^{4}$ coupling with a Higgs propagator, which gives 
a contribution to the amplitude of order

$$
\delta \mathcal{M}\left(Z_{L}^{4} \rightarrow Z_{L}^{4}\right) \sim\left(\frac{\delta_{3} m_{h}^{2}}{v^{3}}\right)^{2} \frac{1}{E^{2}} .
$$

which is parametrically small compared to the contribution that gives the bound eq. (2.23):

$$
\mathcal{M}\left(Z_{L}^{4} \rightarrow Z_{L}^{4}\right) \sim \frac{\left(\delta_{4}-6 \delta_{3}\right) m_{h}^{2}}{v^{6}} .
$$

As noted earlier, it is difficult to experimentally constrain the Higgs quartic interaction even at future colliders, so it is unlikely that one can use eq. (2.23) to give an experimental estimate of the scale of new physics. This is unfortunate, since for generic values of $\delta_{3}, \delta_{4} \lesssim$ 1 , the bound eq. (2.23) is stronger than the model-independent bound eq. (2.20). However, this is not completely model-independent, since the bound eq. (2.23) disappears for $\delta_{4}=$ $6 \delta_{3}$. In fact, this special choice corresponds to having only a $Y^{3}$ coupling, which is natural from a UV point of view; we will discuss this point further below. It also clarifies the large difference between the model-independent bound eq. (2.20), and the stronger bounds obtained when we assumed that only the $h^{3}$ term was modified (see figure 2). Those stronger bounds come from processes with high multiplicity, and these can be cancelled if we allow arbitrary conspiracies among couplings.

So far we have only considered Higgs potential interactions. For completeness, we must also consider the effect of including derivative interactions in our model-independent bound. For example, we can consider the term

$$
\delta \mathcal{L}=\frac{1}{f} X(\partial X)^{2} \supset \frac{1}{f} h(\partial h)^{2} .
$$

which gives a momentum dependent contribution to the Higgs three point function. This mimics a Higgs trilinear $\delta_{3} \sim v / f$ near the threshold for Higgs pair production. However, because of the extra derivatives, this gives rise to unitarity violating processes that grow faster with energy than the potential modifications. For example, $h h \rightarrow Z_{L} Z_{L}$ has a matrix element $\mathcal{M} \sim E^{2} /(f v) \sim \delta_{3} E^{2} / v^{2}$, with a unitarity bound $E \lesssim \sqrt{16 \pi v^{2} /\left|\delta_{3}\right|} \sim$ $2 \mathrm{TeV} / \sqrt{\left|\delta_{3}\right|}$. We see that attempting to explain a large deviation in the Higgs trilinear coupling with derivative couplings results in a unitarity violating scale that is lower than the model-independent bound eq. (2.20), because they give rise to amplitudes that grow faster at high energies.

As a note of caution, we warn that care must be taken when using equations of motion (or equivalently nonlinear field redefinitions) to simplify the Lagrangian [50, 51]. For example, the nonlinear redefinition

$$
X \rightarrow X-\frac{1}{2 f} X^{2}
$$

(equivalent to redefining $h$ ) eliminates the coupling in eq. (2.27) at linear order in the deviation from the SM. However, this redefinition also changes the SM part of the Lagrangian, which will now have interactions that give rise to unitarity violation. For example, the redefinition eq. (2.28) modifies the $h h Z Z$ coupling, and we find an amplitude for $h h \rightarrow Z_{L} Z_{L}$ 
that grows as $E^{2}$. A closely related example is the case of the dimension- 6 operator

$$
\delta \mathcal{L}=\frac{1}{\Lambda^{2}}\left(\partial|H|^{2}\right)^{2} \supset \frac{1}{\Lambda^{2}}(v+h)^{2} \partial h^{2} .
$$

Note that this modifies the Higgs kinetic term as well as generating the coupling eq. (2.27) with $f=\Lambda^{2} / 2 v$. As shown in [38], a nonlinear field transformation

$$
h \rightarrow h-\frac{v^{2}}{\Lambda^{2}}\left(h+\frac{h^{2}}{v}+\frac{h^{3}}{3 v^{2}}\right)
$$

removes to linear order the derivative self-interactions but leads to modifications of the non-derivative Higgs self-interactions (including the Higgs trilinear) and Higgs couplings to $W, Z$ and top quark. After the transformation the matrix elements for the unitarity violating processes are unchanged, but are much easier to see directly by using the original form of the derivative operator.

As a final example, we consider the coupling

$$
\delta \mathcal{L}=\frac{1}{2} \delta Z_{h}(\partial X)^{2}=\frac{1}{2} \delta Z_{h}(\partial h)^{2}+O\left(\vec{G}^{2}\right),
$$

which changes the normalization of the $h$ kinetic term, and therefore the physical Higgs cubic coupling by $\delta_{3} \sim \delta Z_{h}$. It also changes the Higgs couplings to all other SM particles. Because of this there are many additional processes that violate unitarity, but the unitarity violating processes that arise from the modified Higgs cubic are still present, and so these processes cannot change our model-independent bounds. ${ }^{2}$

To summarize the results of this section, a "generic" modification of the low-energy Higgs potential gives rise to unitarity violation at a few $\mathrm{TeV}$. This unitarity violation arises in processes involving many Higgs and gauge particles and $W$ and $Z$ bosons, and these can be canceled if the parameters of the low-energy Higgs potential obey special relations. Allowing for these cancellations, there is still a model-independent bound that depends only on the deviation in the Higgs cubic, but the scale of unitarity violation is much higher $(\sim 13 \mathrm{TeV})$. This scale is potentially accessible to a future $100 \mathrm{TeV} p p$ collider, although it will be more challenging.

\section{Models with a nonstandard Higgs trilinear coupling}

The results of the previous section raise the question of whether the model-independent bound is too conservative. After all, from the bottom-up point of view it appears to require a large number of conspiracies among low-energy parameters to avoid the much stronger

\footnotetext{
${ }^{2}$ The couplings of the Higgs to the other SM fields such as the $W$ and $Z$ bosons are more accurately known to agree with the SM than the Higgs self-coupling, so it would seem that we require $\delta Z_{h} \lesssim 0.1$. However, we can artificially cancel these deviations by adding terms such as

$$
\delta \mathcal{L}=\left(\frac{1}{2} m_{Z}^{2} Z^{\mu} Z_{\mu}+m_{W}^{2} W^{+\mu} W_{\mu}^{-}\right) \frac{h}{v} .
$$

If we do this, we also cancel the growing amplitudes that involve these additional couplings, but the bound from the Higgs trilinear deviation still applies.
} 
bound for the "generic" modification of the Higgs potential. However, we will now show that it is difficult to construct a UV theory with strongly coupled interactions between many Higgs and gauge bosons, as would be required to saturate the "generic" bound. On the other hand, the model-independent bound can be saturated in a UV model with Higgs compositeness at the unitarity violating scale. The model that saturates the bound requires fine-tuning of 2 parameters (compared to 1 parameter in the SM itself), but it gives an existence proof that the model-independent bound can be saturated in a sensible UV theory. If we reduce the tuning in the model, we find that the scale of new physics goes below the unitarity bound, suggesting that the model-independent bound may be too conservative.

We now construct a UV theory that gives rise to the desired modification of the Higgs trilinear, and where the new physical appears at the model-independent unitarity bound eq. (2.20). In such a theory, the couplings of the Higgs must get strong at a scale $M=13.4 \mathrm{TeV} /\left|\delta_{3}\right|^{1 / 2}$, where the new particles enter to unitarize the theory. Because we want the non-derivative interactions in the Higgs potential to become strong at the scale $M$, we do not consider models where the Higgs is a pseudo Nambu-Goldstone boson. If we assume that the strength of the interactions of the Higgs at the scale $M$ is given by a dimensionless coupling $g_{*}$, then the effective Lagrangian below the scale $M$ is given by

$$
\begin{aligned}
\delta \mathcal{L}_{\text {eff }} & =\frac{M^{4}}{g_{*}^{2}} F\left(\frac{g_{*} H}{M}, \frac{\partial}{M}, \ldots\right) \\
& \sim D^{\mu} H^{\dagger} D_{\mu} H+M^{2} H^{\dagger} H+\frac{g_{*}^{2}}{2 !}\left(H^{\dagger} H\right)^{2}+\frac{g_{*}^{4}}{3 ! M^{2}}\left(H^{\dagger} H\right)^{3}+\frac{g_{*}^{2}}{2 ! M^{2}}\left(\partial_{\mu}|H|^{2}\right)^{2}+\cdots .
\end{aligned}
$$

This is a modification of the SILH power counting to the case where the Higgs is not a pseudo-Nambu-Goldstone boson [38, 47]. For $g_{*} \sim 4 \pi / \sqrt{N}$ eq. (3.1) reproduces "naïve dimensional analysis" (NDA) for large- $N$ theories $[52,53]$, which works reasonably well in estimating the size of terms in the effective chiral Lagrangian of QCD, as well as in calculable strongly-coupled SUSY theories [54-56]. The second line in eq. (3.1) should be taken as a rough approximation. For example, the factors of $1 / n$ ! multiplying $\left(H^{\dagger} H\right)^{n}$ can be justified for large $n$, but may be questioned for small values of $n$. We will assume that $M$ is given by our conservative unitarity bound in eq. (2.20), and fix $g_{*}$ from the Higgs trilinear deviation $\delta_{3}$. We obtain $g_{*} \sim 6.9$, independent of $\delta_{3}$. In this model, the effect of higher order terms in the potential at low energies is suppressed compared to the $\left(H^{\dagger} H\right)^{3}$ term by powers of

$$
\left(\frac{g_{*} v}{M}\right)^{2} \sim \frac{1}{60}\left|\delta_{3}\right|
$$

justifying the use of the low-energy expansion in this model. Also, terms involving derivatives are suppressed compared to those without derivatives by powers of $1 / g_{*}$. For example, the operator $\partial^{\mu}\left(H^{\dagger} H\right) \partial_{\mu}\left(H^{\dagger} H\right)$ contributes a contribution to the Higgs trilinear deviation $\delta\left(\delta_{3}\right) / \delta_{3} \sim m_{h}^{2} /\left(g_{*}^{2} v^{2}\right) \sim 0.005$. The difficulty with a model of this kind is that the $H^{\dagger} H$ and $\left(H^{\dagger} H\right)^{2}$ terms are much too large compared to the Standard Model values. There is no 
symmetry difference between the various powers of $H^{\dagger} H$, in eq. (3.1), and it appears that the only way to get agreement with the SM is to fine-tune the $H^{\dagger} H$ and $\left(H^{\dagger} H\right)^{2}$ terms to be small. ${ }^{3}$ The overall tuning is the product of the tuning of the two terms, and is given by

$$
\text { tuning } \sim \frac{\left|m_{H}^{2}\right|}{M^{2}} \frac{\lambda_{H}}{g_{*}^{2}} \sim \frac{\left|\delta_{3}\right|}{5 \times 10^{6}} .
$$

The tuning gets worse for small $\delta_{3}$, because the scale of new physics required to get the deviation of the Higgs cubic becomes larger. The tuning of the $H^{\dagger} H$ term could be explained by anthropic arguments [57], but there is no anthropic reason for the tuning of the $\left(H^{\dagger} H\right)^{2}$ term, so such a model still has an unexplained tuning of order $1 / 500$. In other words, this model does not naturally account for the fact that the Higgs appears to be a weakly-coupled particle at low energies. A model of this kind is not an attractive paradigm for physics beyond the SM, but it does provide an existence proof for models that saturate the model-independent unitarity bound.

If we consider more natural UV models, the scale of new physics is below the modelindependent unitarity bound. For example, we can consider a model of the type eq. (3.1), but with a smaller value of $g_{*}$. Such a model requires a lower value of $M$ to explain a given deviation $\delta_{3}$, simultaneously making the model more natural while lowering the scale of new physics. For example, for $g_{*} \sim 1$ the UV physics is weakly coupled, and the scale of new physics is given by

$$
M \sim \sqrt{\frac{1}{c_{3}}}=\sqrt{\frac{v^{4}}{3 m_{h}^{2} \delta_{3}}}=\frac{280 \mathrm{GeV}}{\sqrt{\left|\delta_{3}\right|}} .
$$

Such a model therefore requires new physics at the electroweak scale, and this kind of new physics is strongly constrained by direct searches, electroweak precision tests, and also obtaining the observed Higgs mass. In fact, as reviewed in [58], in many beyond the Standard Model frameworks (e.g. supersymmetry, composite Higgs) it is difficult to have modifications of the Higgs trilinear larger than $10-20 \%$ due to these constraints. A natural framework for new physics that allows somewhat larger deviations is induced electroweak symmetry breaking [3-6], which also requires new physics below the $\mathrm{TeV}$ scale.

Are there reasonable UV models with new particles at the "generic" unitarity violating scale $E_{\max } \sim 5 \mathrm{TeV}$ (see figure 2), with the correct low energy expansion? This bound arises from processes involving many particles, so the basic requirement is that higher powers of $H$ are suppressed by powers of $v$ with order- 1 coefficients, for example

$$
\delta V \sim \delta_{3} m_{h}^{2} v^{2} \sum_{n=3}^{\infty} a_{n}\left(\frac{H^{\dagger} H}{v^{2}}\right)^{n}, \quad a_{n} \sim 1 .
$$

We want to reproduce this in a UV model where the Higgs is a composite particle with strong interactions at the scale $M$. As previously noted, we want the non-derivative terms

\footnotetext{
${ }^{3}$ There is no sign constraint on the coefficients of the $H^{\dagger} H$ and $\left(H^{\dagger} H\right)^{2}$ terms, so we expect that there are fine-tuned models where their coefficients can be anomalously small for special choices of parameters. For example, a negative $\left(H^{\dagger} H\right)^{2}$ term can arise from the tree-level exchange of a massive singlet scalar field $S$ with a $S H^{\dagger} H$ coupling.
} 
to violate unitarity, so we do not assume that the Higgs is a pseudo Nambu-Goldstone boson. We then expect the potential to be given by the power counting eq. (3.1), which requires $M \sim g_{*} v$. For $M \sim 5 \mathrm{TeV}$, this gives $g_{*} \sim 20$, which is even stronger than the strongest coupling one would expect based on considerations of unitarity or NDA. More problematically, matching the prefactor in the potential requires

$$
\delta_{3} \sim \frac{M^{2}}{3 m_{h}^{2}} \sim 500
$$

Thus, to saturate the generic bound, the Higgs VEV, mass and trilinear must all be tuned to be consistent with current constraints, requiring a total tuning of $\sim 10^{-10}$.

Our conclusion that it is difficult to construct a UV model that generates a "generic" deviation in the Higgs potential. On the other hand, we have shown that if the Higgs is composite it is possible to saturate the weaker model-independent bound. However, even this model is very fine-tuned, and thus we expect the scale of new physics to be below the model-independent bound. For example, induced electroweak symmetry breaking is an existence proof of a class of models that have large deviations in the Higgs trilinear, while giving a natural explanation of the successes of the SM. We believe that these considerations only strengthen the motivation for the measurement of the Higgs cubic.

\section{Conclusions}

We have considered the scales of unitarity violation in a theory where the low-energy Higgs potential is modified from the Standard Model prediction. The Standard Model predicts precise cancellations among different diagrams to guarantee good high energy behavior, and any deviation from the Standard Model predictions for couplings will upset this behavior and lead to the breakdown of perturbation theory at high energies. This is a classic argument that was used to predict the existence of new physics below the $\mathrm{TeV}$ scale in the theory without a Higgs sector, providing a "no lose" theorem for the LHC.

We extended this argument to a theory with a Higgs, but with modifications of the Higgs potential. Using the equivalence theorem, we can determine which processes involving longitudinal $W$ 's and $Z$ 's and Higgs particles violate unitarity, and easily compute their high-energy behavior. We have shown that generic modifications of the Higgs trilinear coupling lead to the theory breaking down near $5 \mathrm{TeV}$, nearly independently of the size of the deviation. This scale is tantalizingly close to the energy scale currently being probed by the LHC. However, the bad high energy behavior can be canceled by deviations in higher order terms in the Higgs potential, and is therefore not model-independent. We find that there is a completely model-independent bound on the scale of new physics that depends only on the modification of the Higgs trilinear coupling at low energies: the theory must break down at a scale $\lesssim 13 \mathrm{TeV} /\left|\delta_{3}\right|$, where $\delta_{3}$ is the fractional modification of the Higgs trilinear coupling. This means that measurements of the Higgs trilinear directly point to a new UV energy scale where new physics must appear, giving additional motivation for these searches. If any deviation is observed, it would provide a target for future high energy colliders designed to explore this higher energy scale. 
The growing amplitudes discussed in this work motivate experimental searches in these channels. Much of the existing work on searching for new high energy physics in electroweak final states has focused on low multiplicity final states, such as $Z h$ [59], $t W$ [60], and $(t Z, t h)+$ jet [61]. A recent paper [40] has considered amplitudes whose energy growth results from Higgs coupling modifications. One of their analyses probed $V V \rightarrow W W h(V=$ $W$ or $Z$ ) using vector boson fusion, and found sensitivity to $\left|\delta_{3}\right| \gtrsim 5$ at the high luminosity LHC. Given our analysis, it would be interesting to explore unitarity systematically for other processes such as $V V \rightarrow V V V V$.

\section{Acknowledgments}

We thank A. Falkowski and R. Rattazzi for discussions about their closely related paper [49]. We especially thank E. Salvioni for extensive discussions and comments on the draft. SC is supported in part by the U.S. Department of Energy under grant DESC0011640. ML is supported in part by the U.S. Department of Energy under grant DE-SC-0009999.

\section{A Unitarity bound on potential interactions}

We are interested in the bounds placed on tree-level scattering amplitudes by unitarity. The idea of these unitarity constraints is very simple. We write the $S$ matrix as

$$
S=\mathbb{1}+i T
$$

where the identity contribution represents the free propagation of particles without interactions, and the transition matrix $T$ describes interactions. Unitarity of the $S$ matrix implies that if $|i\rangle$ and $|f\rangle$ are unit normalized states we have

$$
|\langle f|S| i\rangle| \leq 1 \quad \text { for all } i, j .
$$

For $|f\rangle \neq|i\rangle$, this implies

$$
|\langle f|T| i\rangle| \leq 1
$$

Plane-wave states are not unit normalized, but we can define normalized states using the partial wave expansion. More generally, we can label the initial and final states by the total 4-momentum $P^{\mu}$, and we assume that the additional quantum numbers $\alpha$ required to specify the state are discrete, so that the states are normalized to

$$
\left\langle P^{\prime}, \alpha^{\prime} \mid P, \alpha\right\rangle=(2 \pi)^{4} \delta^{4}\left(P-P^{\prime}\right) \delta_{\alpha \alpha^{\prime}} .
$$

For example, in the partial wave expansion of a state of 2 scalar particles, we can take $\alpha$ to consist of the relative angular momentum quantum numbers $\ell$ and $m$. Defining the Lorentz invariant amplitude

$$
\left\langle P^{\prime}, \alpha^{\prime}|T| P, \alpha\right\rangle=(2 \pi)^{4} \delta^{4}\left(P-P^{\prime}\right) \mathcal{M}_{\alpha^{\prime} \alpha},
$$


we have

$$
\left\langle P_{f}, \beta|S| P_{i}, \alpha\right\rangle=(2 \pi)^{4} \delta^{4}\left(P_{f}-P_{i}\right) S_{\beta \alpha},
$$

with

$$
S_{\beta \alpha}=\delta_{\beta \alpha}+i \mathcal{M}_{\beta \alpha}
$$

Unitarity of the $S$-matrix implies that the matrix $S_{\beta \alpha}$ is unitary, and the same logic as above implies that

$$
\left|\mathcal{M}_{\beta \alpha}\right| \leq 1
$$

for $\beta \neq \alpha$. For a bound when $\beta=\alpha$, consider

$$
1=\delta_{\alpha \alpha}=\sum_{\gamma} S_{\gamma \alpha}^{*} S_{\gamma \alpha}=1-2 \operatorname{Im} \mathcal{M}_{\alpha \alpha}+\sum_{\gamma}\left|\mathcal{M}_{\gamma \alpha}\right|^{2}
$$

which gives

$$
2 \operatorname{Im} \mathcal{M}_{\alpha \alpha}=\sum_{\gamma}\left|\mathcal{M}_{\gamma \alpha}\right|^{2} \geq\left|\mathcal{M}_{\alpha \alpha}\right|^{2}=\left|\operatorname{Re} \mathcal{M}_{\alpha \alpha}\right|^{2}+\left|\operatorname{Im} \mathcal{M}_{\alpha \alpha}\right|^{2}
$$

Completing the square shows that $1 \geq\left|\operatorname{Re} \mathcal{M}_{\alpha \alpha}\right|^{2}+\left|\operatorname{Im} \mathcal{M}_{\alpha \alpha}-1\right|^{2}$. This implies the bounds

$$
\left|\operatorname{Re} \mathcal{M}_{\alpha \alpha}\right| \leq 1, \quad 0 \leq \operatorname{Im} \mathcal{M}_{\alpha \alpha} \leq 2
$$

Since $\mathcal{M}_{\alpha \alpha}$ is real at tree level, this implies that $\left|\mathcal{M}_{\alpha \beta}\right| \leq 1$ is true at tree level for all states $\alpha, \beta$.

Let us apply these ideas to obtain the unitarity bound on an effective interaction term of the form

$$
\mathcal{L}_{\text {int }}=\frac{\lambda_{n}}{n_{1} ! \cdots n_{r} !} \phi_{1}^{n_{1}} \phi_{2}^{n_{2}} \cdots \phi_{r}^{n_{r}}
$$

where $\phi_{i}$ are independent, real scalar fields and $n_{i}$ are positive integers. We define the normalized states

$$
\left|P, k_{1}, \ldots, k_{r}\right\rangle=C_{k_{1} \cdots k_{r}} \int d^{4} x e^{i P \cdot x} \prod_{i=1}^{r}\left[\phi_{i}^{(-)}(x)\right]^{k_{i}}|0\rangle,
$$

where the $k_{i}$ are non-negative integers that play the role of the discrete label $\alpha$ in eq. (A.4), and $\phi_{i}^{(-)}$is the part of the (interaction picture) field $\phi_{i}$ that contains a creation operator. The idea behind the states in eq. (A.13) is that they have the largest overlap with the interaction eq. (A.12), and will therefore give the strongest unitarity bounds. The normalization eq. (A.4) then fixes

$$
\frac{1}{\left|C_{k_{1} \cdots k_{r}}\right|^{2}}=\frac{1}{(k-1) !(k-2) !} \frac{1}{8 \pi}\left(\prod_{i=1}^{r} k_{i} !\right)\left(\frac{E}{4 \pi}\right)^{2 k-4}
$$


where $k=k_{1}+\cdots+k_{r}$ and we have assumed all the particles are massless. Working out the matrix element for the scattering amplitude with $k_{i} \rightarrow n_{i}-k_{i}$, we get

$$
\begin{aligned}
\mathcal{M}_{n-k, k}= & \frac{\lambda_{n}}{n_{1} ! \cdots n_{r} !} \frac{1}{C_{n_{1}-k_{1} \cdots n_{r}-k_{r} C_{k_{1} \cdots k_{r}}^{*}}} \prod_{i=1}^{r}\left(\begin{array}{c}
n_{i} \\
k_{i}
\end{array}\right) \\
= & \frac{\lambda_{n}}{8 \pi \sqrt{(k-1) !(k-2) !(n-k-1) !(n-k-2) !}}\left(\frac{E}{4 \pi}\right)^{n-4} \\
& \times \prod_{i=1}^{r}\left(\begin{array}{c}
n_{i} \\
k_{i}
\end{array}\right) \frac{\sqrt{k_{i} !\left(n_{i}-k_{i}\right) !}}{n_{i} !}
\end{aligned}
$$

where $n=n_{1}+\cdots+n_{r}$. Requiring this to be less than 1 gives the bound

$$
E_{k} \leq 4 \pi\left(\frac{8 \pi \prod_{i=1}^{r} n_{i} !}{\lambda_{n} \prod_{i=1}^{r}\left(\begin{array}{l}
n_{i} \\
k_{i}
\end{array}\right)}\right)^{1 /(n-4)}\left(\frac{(k-1) !(k-2) !(n-k-1) !(n-k-2) !}{\prod_{i=1}^{r} k_{i} !\left(n_{i}-k_{i}\right) !}\right)^{1 /(2 n-8)}
$$

The lowest unitarity limit is when $k_{i}=\frac{1}{2} n_{i}$ (assuming all the $n_{i}$ are even), which improves on the conventionally analyzed $2 \rightarrow m$ scattering processes. This gives

$$
E_{k=n / 2} \leq 4 \pi\left(\frac{8 \pi\left(\frac{n}{2}-1\right) !\left(\frac{n}{2}-2\right) ! \prod_{i=1}^{r}\left(n_{i} / 2\right) !}{\lambda_{n}}\right)^{1 /(n-4)} .
$$

In the large $n$ limit, the bound asymptotically gets worse, although intermediate multiplicities may still give better bounds since it can counteract the values of $\lambda_{n}$ in a given model.

Using this formula, we can determine the optimal channel (i.e. choice of $k_{1}, \ldots, k_{r}$ ) to get the lowest bound. For a representative set of five to eight point interactions, in table 2 we list the optimal energy bound and the channel it can come from (there are multiple choices coming from permutations and swapping of initial and final states). We note that in the above, we have neglected contributions to scattering amplitudes that involve multiple insertions of the interactions. These involve diagrams with one or more internal propagator, and this means that these interactions scale with energy with a power less than that of the model-dependent terms.

For interactions with many correlated couplings, an improved unitarity bound can be found by diagonalizing the transition matrix element. For example, for the six point interaction

$$
\frac{m_{h}^{2}}{16 v^{4}} \delta_{3} \vec{G}^{6}=\frac{m_{h}^{2}}{16 v^{4}} \delta_{3}\left(G_{1}^{2}+G_{2}^{2}+G_{3}^{2}\right)^{3},
$$

we expect the best scattering channel to appear for a specific custodial $S U(() 2)$ representation. Focusing on the 3 to 3 processes, we use the basis

$$
(\{3,0,0\}\{0,3,0\}\{0,0,3\}\{2,1,0\}\{2,0,1\}\{1,2,0\}\{0,2,1\}\{1,0,2\}\{0,1,2\}\{1,1,1\})^{T}
$$




\begin{tabular}{|l|l|c|}
\hline$\left(n_{1}, \ldots, n_{r}\right)$ & Best $\left(k_{1}, \ldots, k_{r}\right)$ & $E_{\max }$ \\
\hline$(5)$ & $(2)$ & $1550 / \lambda_{n}$ \\
$(4,1)$ & $(2,0)$ & $893 / \lambda_{n}$ \\
$(3,2)$ & $(1,1)$ & $632 / \lambda_{n}$ \\
$(3,1,1)$ & $(1,1,0)$ & $632 / \lambda_{n}$ \\
$(2,2,1)$ & $(1,1,0)$ & $447 / \lambda_{n}$ \\
$(2,1,1,1)$ & $(1,1,0,0)$ & $447 / \lambda_{n}$ \\
\hline$(6)$ & $(3)$ & $218 / \sqrt{\lambda_{n}}$ \\
$(5,1)$ & $(2,1)$ & $166 / \sqrt{\lambda_{n}}$ \\
$(4,2)$ & $(2,1)$ & $126 / \sqrt{\lambda_{n}}$ \\
$(3,3)$ & $(2,1)$ & $126 / \sqrt{\lambda_{n}}$ \\
$(4,1,1)$ & $(2,1,0)$ & $126 / \sqrt{\lambda_{n}}$ \\
$(3,2,1)$ & $(1,1,1)$ & $106 / \sqrt{\lambda_{n}}$ \\
$(2,2,2)$ & $(1,1,1)$ & $89 / \sqrt{\lambda_{n}}$ \\
$(3,1,1,1)$ & $(1,1,1,0)$ & $106 / \sqrt{\lambda_{n}}$ \\
$(2,2,1,1)$ & $(1,1,1,0)$ & $89 / \sqrt{\lambda_{n}}$ \\
\hline$(7)$ & $(3)$ & $143 / \lambda_{n}^{1 / 3}$ \\
$(6,1)$ & $(3,0)$ & $114 / \lambda_{n}^{1 / 3}$ \\
$(4,2,1)$ & $(2,1,1)$ & $79 / \lambda_{n}^{1 / 3}$ \\
$(2,2,2,1)$ & $(1,1,1,0)$ & $63 / \lambda_{n}^{1 / 3}$ \\
\hline$(8)$ & $(4)$ & $116 / \lambda_{n}^{1 / 4}$ \\
$(6,2)$ & $(3,1)$ & $82 / \lambda_{n}^{1 / 4}$ \\
$(4,4)$ & $(2,2)$ & $74 / \lambda_{n}^{1 / 4}$ \\
$(4,2,2)$ & $(2,1,1)$ & $62 / \lambda_{n}^{1 / 4}$ \\
\hline
\end{tabular}

Table 2. The lowest unitarity violating energy scales for some five to eight point interactions of the form eq. (A.12), with a representative process that gives the stated bound.

where $\left\{n_{1}, n_{2}, n_{3}\right\}$ represents the number of goldstones $\left\{G_{1}, G_{2}, G_{3}\right\}$ in the state. The transition matrix is

$$
\left(\begin{array}{cccccccccc}
5 & 0 & 0 & 0 & 0 & \sqrt{3} & 0 & \sqrt{3} & 0 & 0 \\
0 & 5 & 0 & \sqrt{3} & 0 & 0 & 0 & 0 & \sqrt{3} & 0 \\
0 & 0 & 5 & 0 & \sqrt{3} & 0 & \sqrt{3} & 0 & 0 & 0 \\
0 & \sqrt{3} & 0 & 3 & 0 & 0 & 0 & 0 & 1 & 0 \\
0 & 0 & \sqrt{3} & 0 & 3 & 0 & 1 & 0 & 0 & 0 \\
\sqrt{3} & 0 & 0 & 0 & 0 & 3 & 0 & 1 & 0 & 0 \\
0 & 0 & \sqrt{3} & 0 & 1 & 0 & 3 & 0 & 0 & 0 \\
\sqrt{3} & 0 & 0 & 0 & 0 & 1 & 0 & 3 & 0 & 0 \\
0 & \sqrt{3} & 0 & 1 & 0 & 0 & 0 & 0 & 3 & 0 \\
0 & 0 & 0 & 0 & 0 & 0 & 0 & 0 & 0 & 2
\end{array}\right) \text { } 3 m_{h}^{2} \delta_{3} E^{2}
$$

which can be diagonalized to get a matrix with eigenvalues $7 \cdot \frac{3 m_{h}^{2} \delta_{3}}{512 \pi^{3} v^{4}} E^{2}, 2 \cdot \frac{3 m_{h}^{2} \delta_{3}}{512 \pi^{3} v^{4}} E^{2}$ with multiplicity 3 and 7 respectively, which are the $I=1,3$ scattering channels. Utilizing 
the larger eigenvalue for the $I=1$ channel leads to an optimized unitarity bound for 3 $G$ to $3 G$ scattering of $13.4 \mathrm{TeV} / \sqrt{\left|\delta_{3}\right|}$. A similar analysis can also be performed for the $h \vec{G}^{4}$ interaction. Analyzing the allowed 2 to 3 transition matrix, one finds eigenvalues of $\frac{15 m_{h}^{2} \delta_{3}}{64 \sqrt{2} \pi^{2} v^{3}} E, \frac{3 \sqrt{5} m_{h}^{2} \delta_{3}}{64 \pi^{2} v^{3}} E$, and $\frac{3 m_{h}^{2} \delta_{3}}{32 \sqrt{2} \pi^{2} v^{3}} E$ for the $I=0,1,2$ channels. The best unitarity bound of $57.4 \mathrm{TeV} /\left|\delta_{3}\right|$ comes from the $I=0$ channel.

Open Access. This article is distributed under the terms of the Creative Commons Attribution License (CC-BY 4.0), which permits any use, distribution and reproduction in any medium, provided the original author(s) and source are credited.

\section{References}

[1] ATLAS collaboration, Combined measurements of Higgs boson production and decay using up to $80 \mathrm{fb}^{-1}$ of proton-proton collision data at $\sqrt{s}=13 \mathrm{TeV}$ collected with the ATLAS experiment, ATLAS-CONF-2018-031 (2018).

[2] CMS collaboration, Combined measurements of Higgs boson couplings in proton-proton collisions at $\sqrt{s}=13 \mathrm{TeV}$, Eur. Phys. J. C 79 (2019) 421 [arXiv:1809.10733] [InSPIRE].

[3] A. Azatov, J. Galloway and M.A. Luty, Superconformal technicolor, Phys. Rev. Lett. 108 (2012) 041802 [arXiv: 1106.3346] [INSPIRE].

[4] A. Azatov, J. Galloway and M.A. Luty, Superconformal technicolor: models and phenomenology, Phys. Rev. D 85 (2012) 015018 [arXiv:1106.4815] [INSPIRE].

[5] J. Galloway, M.A. Luty, Y. Tsai and Y. Zhao, Induced electroweak symmetry breaking and supersymmetric naturalness, Phys. Rev. D 89 (2014) 075003 [arXiv:1306.6354] [INSPIRE].

[6] S. Chang et al., Phenomenology of induced electroweak symmetry breaking, JHEP 03 (2015) 017 [arXiv: 1411.6023] [INSPIRE].

[7] C. Grojean, G. Servant and J.D. Wells, First-order electroweak phase transition in the standard model with a low cutoff, Phys. Rev. D 71 (2005) 036001 [hep-ph/0407019] [INSPIRE].

[8] ATLAS collaboration, Combination of searches for Higgs boson pairs in pp collisions at $\sqrt{s}=13 \mathrm{TeV}$ with the ATLAS detector, Phys. Lett. B 800 (2020) 135103 [arXiv: 1906. 02025] [INSPIRE].

[9] ATLAS collaboration, Constraints on the Higgs boson self-coupling from the combination of single-Higgs and double-Higgs production analyses performed with the ATLAS experiment, ATLAS-CONF-2019-049 (2019).

[10] CMS collaboration, Combination of searches for Higgs boson pair production in proton-proton collisions at $\sqrt{s}=13$ TeV, Phys. Rev. Lett. 122 (2019) 121803 [arXiv: 1811.09689] [INSPIRE].

[11] ATLAS collaboration, Study of the double Higgs production channel $H(\rightarrow b \bar{b}) H(\rightarrow \gamma \gamma)$ with the ATLAS experiment at the HL-LHC, ATL-PHYS-PUB-2017-001 (2017).

[12] CMS collaboration, Higgs pair production at the High Luminosity LHC, CMS-PAS-FTR-15-002 (2015).

[13] M. Cepeda et al., Report from Working Group 2, CERN Yellow Rep. Monogr. 7 (2019) 221 [arXiv: 1902.00134] [INSPIRE]. 
[14] C.F. Dürig, Measuring the Higgs self-coupling at the international linear collider, Ph.D. thesis, Hamburg University, Hamburg, Germany (2016) [DESY-THESIS-2016-027].

[15] H. Abramowicz et al., Higgs physics at the CLIC electron-positron linear collider, Eur. Phys. J. C 77 (2017) 475 [arXiv:1608.07538] [INSPIRE].

[16] D. Gonçalves et al., Higgs boson pair production at future hadron colliders: from kinematics to dynamics, Phys. Rev. D 97 (2018) 113004 [arXiv:1802.04319] [inSPIRE].

[17] A. Papaefstathiou and K. Sakurai, Triple Higgs boson production at a $100 \mathrm{TeV}$ proton-proton collider, JHEP 02 (2016) 006 [arXiv:1508.06524] [INSPIRE].

[18] C.-Y. Chen et al., Probing triple-Higgs productions via $4 b 2 \gamma$ decay channel at a $100 \mathrm{TeV}$ hadron collider, Phys. Rev. D 93 (2016) 013007 [arXiv:1510.04013] [INSPIRE].

[19] A.S. Belyaev, P.B. Schaefers and M.C. Thomas, Precise test of Higgs boson properties via triple Higgs boson production in vector boson fusion at future colliders, Phys. Rev. D 99 (2019) 015030 [arXiv: 1801.10157] [INSPIRE].

[20] S. Dawson, C. Englert and T. Plehn, Higgs physics: it ain't over till it's over, Phys. Rept. 816 (2019) 1 [arXiv: 1808.01324] [inSPIRE].

[21] G. Degrassi, M. Fedele and P.P. Giardino, Constraints on the trilinear Higgs self coupling from precision observables, JHEP 04 (2017) 155 [arXiv: 1702.01737] [INSPIRE].

[22] G.D. Kribs et al., Electroweak oblique parameters as a probe of the trilinear Higgs boson self-interaction, Phys. Rev. D 95 (2017) 093004 [arXiv: 1702.07678] [INSPIRE].

[23] M. McCullough, An indirect model-dependent probe of the Higgs self-coupling, Phys. Rev. D 90 (2014) 015001 [Erratum ibid. D 92 (2015) 039903] [arXiv: 1312.3322] [INSPIRE].

[24] M. Gorbahn and U. Haisch, Indirect probes of the trilinear Higgs coupling: gg $\rightarrow h$ and $h \rightarrow \gamma \gamma$, JHEP 10 (2016) 094 [arXiv: 1607.03773] [INSPIRE].

[25] G. Degrassi, P.P. Giardino, F. Maltoni and D. Pagani, Probing the Higgs self coupling via single Higgs production at the LHC, JHEP 12 (2016) 080 [arXiv: 1607.04251] [INSPIRE].

[26] T. Liu, K.-F. Lyu, J. Ren and H.X. Zhu, Probing the quartic Higgs boson self-interaction, Phys. Rev. D 98 (2018) 093004 [arXiv: 1803.04359] [InSPIRE].

[27] W. Bizon, U. Haisch and L. Rottoli, Constraints on the quartic Higgs self-coupling from double-Higgs production at future hadron colliders, JHEP 10 (2019) 267 [arXiv:1810.04665] [INSPIRE].

[28] S. Borowka et al., Probing the scalar potential via double Higgs boson production at hadron colliders, JHEP 04 (2019) 016 [arXiv: 1811.12366] [INSPIRE].

[29] B.W. Lee, C. Quigg and H.B. Thacker, Weak interactions at very high-energies: the role of the Higgs boson mass, Phys. Rev. D 16 (1977) 1519 [InSPIRE].

[30] B.W. Lee, C. Quigg and H.B. Thacker, Strength of weak interactions at very high energies and the higgs boson mass, Phys. Rev. Lett. 38 (1977) 883.

[31] D.A. Dicus and V.S. Mathur, Upper bounds on the values of masses in unified gauge theories, Phys. Rev. D 7 (1973) 3111 [INSPIRE].

[32] M.S. Chanowitz and M.K. Gaillard, The TeV physics of strongly interacting $W$ 's and $Z$ 's, Nucl. Phys. B 261 (1985) 379 [InSPIRE]. 
[33] W. Buchmüller and D. Wyler, Effective Lagrangian analysis of new interactions and flavor conservation, Nucl. Phys. B 268 (1986) 621 [INSPIRE].

[34] B. Grzadkowski, M. Iskrzynski, M. Misiak and J. Rosiek, Dimension-six terms in the standard model lagrangian, JHEP 10 (2010) 085 [arXiv: 1008.4884] [INSPIRE].

[35] B. Grinstein and M. Trott, A Higgs-Higgs bound state due to new physics at a TeV, Phys. Rev. D 76 (2007) 073002 [arXiv:0704.1505] [INSPIRE].

[36] LHC Higgs Cross Section Working Group collaboration, Handbook of LHC Higgs cross sections: 4. Deciphering the nature of the Higgs sector, arXiv:1610.07922 [INSPIRE].

[37] I. Brivio and M. Trott, The standard model as an effective field theory, Phys. Rept. 793 (2019) 1 [arXiv: 1706. 08945] [INSPIRE].

[38] G.F. Giudice, C. Grojean, A. Pomarol and R. Rattazzi, The strongly-interacting light Higgs, JHEP 06 (2007) 045 [hep-ph/0703164] [INSPIRE].

[39] A. Belyaev, A.C.A. Oliveira, R. Rosenfeld and M.C. Thomas, Multi Higgs and vector boson production beyond the standard model, JHEP 05 (2013) 005 [arXiv:1212.3860] [INSPIRE].

[40] B. Henning, D. Lombardo, M. Riembau and F. Riva, Measuring Higgs couplings without Higgs bosons, Phys. Rev. Lett. 123 (2019) 181801 [arXiv:1812.09299] [INSPIRE].

[41] L. Di Luzio, R. Gröber and M. Spannowsky, Maxi-sizing the trilinear Higgs self-coupling: how large could it be?, Eur. Phys. J. C 77 (2017) 788 [arXiv:1704.02311] [InSPIRE].

[42] T. Corbett, O.J.P. Éboli and M.C. Gonzalez-Garcia, Unitarity constraints on dimension-six operators, Phys. Rev. D 91 (2015) 035014 [arXiv:1411.5026] [InSPIRE].

[43] T. Corbett, O.J.P. É boli and M.C. Gonzalez-Garcia, Unitarity constraints on dimension-six operators II: including fermionic operators, Phys. Rev. D 96 (2017) 035006 [arXiv: 1705. 09294] [INSPIRE].

[44] J. Jaeckel and V.V. Khoze, Upper limit on the scale of new physics phenomena from rising cross sections in high multiplicity Higgs and vector boson events, Phys. Rev. D 91 (2015) 093007 [arXiv: 1411.5633] [INSPIRE].

[45] V.V. Khoze and M. Spannowsky, Higgsplosion: solving the hierarchy problem via rapid decays of heavy states into multiple Higgs bosons, Nucl. Phys. B 926 (2018) 95 [arXiv: 1704.03447] [INSPIRE].

[46] A. Monin, Inconsistencies of higgsplosion, arXiv:1808.05810 [INSPIRE].

[47] A. Azatov, R. Contino, G. Panico and M. Son, Effective field theory analysis of double Higgs boson production via gluon fusion, Phys. Rev. D 92 (2015) 035001 [arXiv:1502.00539] [INSPIRE].

[48] D. Liu, A. Pomarol, R. Rattazzi and F. Riva, Patterns of strong coupling for LHC searches, JHEP 11 (2016) 141 [arXiv: 1603.03064] [INSPIRE].

[49] A. Falkowski and R. Rattazzi, Which EFT, JHEP 10 (2019) 255 [arXiv:1902.05936] [INSPIRE].

[50] H. Georgi, On-shell effective field theory, Nucl. Phys. B 361 (1991) 339 [INSPIRE].

[51] C. Arzt, Reduced effective Lagrangians, Phys. Lett. B 342 (1995) 189 [hep-ph/9304230] [INSPIRE]. 
[52] A. Manohar and H. Georgi, Chiral quarks and the nonrelativistic quark model, Nucl. Phys. B 234 (1984) 189 [INSPIRE].

[53] H. Georgi and L. Randall, Flavor conserving CP-violation in invisible axion models, Nucl. Phys. B 276 (1986) 241 [INSPIRE].

[54] M.A. Luty, Naive dimensional analysis and supersymmetry, Phys. Rev. D 57 (1998) 1531 [hep-ph/9706235] [INSPIRE].

[55] A.G. Cohen, D.B. Kaplan and A.E. Nelson, Counting 4 pis in strongly coupled supersymmetry, Phys. Lett. B 412 (1997) 301 [hep-ph/9706275] [INSPIRE].

[56] M.A. Luty and R. Rattazzi, Soft supersymmetry breaking in deformed moduli spaces, conformal theories and $N=2$ Yang-Mills theory, JHEP 11 (1999) 001 [hep-th/9908085] [INSPIRE].

[57] V. Agrawal, S.M. Barr, J.F. Donoghue and D. Seckel, Viable range of the mass scale of the standard model, Phys. Rev. D 57 (1998) 5480 [hep-ph/9707380] [INSPIRE].

[58] R.S. Gupta, H. Rzehak and J.D. Wells, How well do we need to measure the Higgs boson mass and self-coupling?, Phys. Rev. D 88 (2013) 055024 [arXiv: 1305.6397] [INSPIRE].

[59] S. Banerjee, C. Englert, R.S. Gupta and M. Spannowsky, Probing electroweak precision physics via boosted Higgs-strahlung at the LHC, Phys. Rev. D 98 (2018) 095012 [arXiv: 1807.01796] [INSPIRE].

[60] J.A. Dror, M. Farina, E. Salvioni and J. Serra, Strong tW scattering at the LHC, JHEP 01 (2016) 071 [arXiv: 1511.03674] [INSPIRE].

[61] C. Degrande et al., Single-top associated production with a $Z$ or $H$ boson at the LHC: the SMEFT interpretation, JHEP 10 (2018) 005 [arXiv: 1804.07773] [INSPIRE]. 